\title{
Whole-body MRI Imaging Is an Essential Tool in Diagnosing and Monitoring Patients With Sterile Osteomyelitis
}
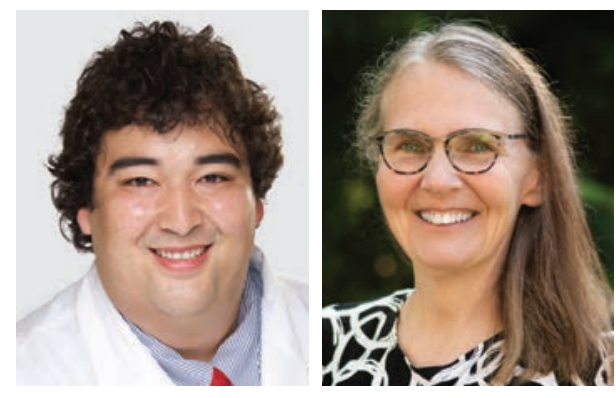

\author{
Takashi Shawn Sato ${ }^{1}$ (i) and Polly J. Ferguson ${ }^{2}$ (i)
}

From the first description in 1972 as "subacute and chronic recurrent osteomyelitis" to the currently recognized chronic recurrent multifocal osteomyelitis (CRMO) or chronic nonbacterial osteitis (CNO), diagnosis and monitoring of patients with this disease has been and continues to be a challenge ${ }^{1,2}$. While the most common presenting symptom is focal bone pain, its waxing and waning nature tends to contribute to the diagnostic odyssey that many patients must endure. Objective changes on examination such as swelling and tenderness over a lesion may not be present or may mimic inflammatory arthritis. Laboratory findings are equally nonspecific, with some patients having a mildly elevated C-reactive protein and/or erythrocyte sedimentation rate, while most other laboratory findings remain normal ${ }^{3}$. In about one-quarter of patients, a comorbid inflammatory condition such as psoriasis or inflammatory bowel disease, when present, often provides the vital clue to establishing a diagnosis ${ }^{4}$. However, in those with osseous involvement only, the lack of specific findings makes the diagnosis of CNO challenging, with patients averaging 2 years between initially presenting with symptoms and receiving a diagnosis of $\mathrm{CNO}^{5}$. Given the lack of pathognomonic features in most patients, a high index of suspicion for $\mathrm{CNO}$ and close collaboration between clinicians and radiologists are important to making a timely diagnosis.

While imaging is essential in establishing a diagnosis of $\mathrm{CNO}$, imaging features of $\mathrm{CNO}$ can also be relatively nonspecific. Plain films lack sensitivity, especially early in the disease

TSS was supported by the University of Iowa's Department of Radiology and Divisions of Pediatric Radiology and Neuroradiology. PJF was supported by NIH/NIAMS R01AR059703 and The Marjorie K. Lamb Professorship at the University of Iowa Carver College of Medicine.

${ }^{I}$ T.S. Sato, Clinical Assistant Professor, Divisions of Pediatric Radiology and Neuroradiology, Department of Radiology, Carver College of Medicine,

University of Iowa; ${ }^{2}$ P.J. Ferguson, Professor, Division of Pediatric

Rheumatology, Allergy and Immunology, Department of Pediatrics, Carver College of Medicine, University of Iowa, Iowa City, Iowa, USA.

Address correspondence to Dr. P. Ferguson, University of Iowa, 200 Hawkins Dr., Iowa City, IA 52240, USA. Email:polly-ferguson@uiowa.edu. course and may be completely normal despite significant disease activity. When positive, plain films demonstrate mixed lytic and sclerotic lesions, most common in the metaphyses of long bones in the lower extremities and mimicking radiologic features of infectious osteomyelitis. Magnetic resonance imaging (MRI) of the painful area is more sensitive for the early findings of $\mathrm{CNO}$, but even with MRI, the findings of marrow edema on T2 or short-tau inversion recovery (STIR) sequences are not specific to this disease process ${ }^{6}$.

When a patient presents with CRMO, although they often present with just a single site of pain, multifocal disease is often present. Whole-body imaging can identify additional asymptomatic or minimally symptomatic lesions, aiding in making a diagnosis of $\mathrm{CRMO}^{7}$. While bone scans can provide wholebody imaging, compared to whole-body MRI (WB-MRI), they require radiation and have decreased sensitivity, spatial resolution, and limited ability to evaluate physeal disease, making WB-MRI superior to bone scan in delineating the extent of disease.

Given that symptomatology is often discordant with radiologic findings, it is important to understand whole-body disease burden not only at diagnosis but also when making treatment decisions $^{2}$. Further, patients with $\mathrm{CNO}$ often need long-term surveillance imaging, and WB-MRI provide an excellent radiation-free tool for monitoring disease.

When performing WB-MRI, it is important to have a tailored examination for the patient population and disease process you are evaluating. In the case of children with $\mathrm{CNO}$, it is important to have a relatively short scan time to eliminate or at least minimize the necessity for sedation. A full-sequence WB-MRI may take 4-6 hours, which is not realistically feasible in this patient population ${ }^{8}$. STIR sequences are relatively fast sequences that are sensitive to the marrow edema seen in $\mathrm{CNO}^{9}$. Many CNO WB-MRI imaging protocols include STIR sequences only, whereas others also include diffusion and/or T1-weighted imaging ${ }^{10,11,12}$. Protocol designs for WB-MRI need

\section{See WB-MRI Scoring in CNO, page 751}


to take into account typical disease distribution. For example, in patients with $\mathrm{CNO}$, it is helpful to cover the whole body in a coronal plane, but additional planes such as sagittal spine, axial pelvis, and sagittal ankle/feet are needed for better lesion visualization in these regions ${ }^{11}$. At our institution, we have created a STIR-based CNO screening/monitoring WB-MRI protocol that can be performed in a single 40-minute MRI time slot, with approximately 25 minutes of MRI scan time. No single acquisition takes longer than 3 minutes, and sedation is rarely necessary (Table 1).

When reviewing a WB-MRI protocol, it is important to understand the potential shortcomings as well. CNO screening/ monitoring WB-MRI protocols based only on STIR sacrifice additional sequences for speed of the exam. Because of this, if there are unexpected or atypical findings, the patients may have to return for a more focused full-sequence regional MRI for complete characterization. At our institution, this rarely must be performed; however, it is important for both the clinician and the radiologists to have an understanding of the strengths and limitations of this type of WB-MRI examination. Despite these limitations, WB-MRI has become the standard for diagnosing and monitoring patients with $\mathrm{CNO}$, with a significantly increased sensitivity, improved anatomical detail, and lack of radiation compared to other whole-body imaging (bone scans and skeletal surveys).

The best treatment for $\mathrm{CNO}$ remains obscure. Zhao, et al surveyed pediatric rheumatologists who participate in the Childhood Arthritis and Rheumatology Research Alliance (CARRA) and found wide variation in how children with $\mathrm{CNO}$ are treated ${ }^{13}$. One reason is because of the low-quality evidence available to guide treatment decisions. Most data are retrospective from case series with just a few prospective studies and no randomized trials. And while WB-MRI has become a vital tool in the clinical care of patients with $\mathrm{CNO}$, standardization in WB-MRI acquisition and interpretation is needed to be able to use imaging as an outcome measure in research, such as in gauging response to therapy in a drug trial.

In this issue of The Journal of Rheumatology, Panwar, et al present a WB-MRI scoring tool for $\mathrm{CNO}$ that was then used to determine treatment response to pamidronate in a retrospec- tive study of pediatric patients with $\mathrm{CNO}^{14}$. They identified 32 patients that met the Bristol Criteria and/or had a bone biopsy consistent with $\mathrm{CNO}$ in which serial WB-MRI were performed at defined intervals pre- and posttreatment with pamidronate (average period of 5 months after each pamidronate cycle and a total of 88 WB-MRI studied). These patients had failed nonsteroidal antiinflammatory drug therapy and were treated with intravenous pamidronate at a dose of $1 \mathrm{mg} / \mathrm{kg} /$ day $(\max 60 \mathrm{mg})$ once per month for 3 months (defined as 1 pamidronate cycle). Two blinded radiologists reviewed the 88 scans using their WB-MRI scoring tool, with excellent interreader reliability of $x$ ranging from 0.93 to 1.00 . Using this tool, they found that 34\% of CNO lesions resolved after a single cycle of pamidronate, while in a subset of 11 patients that required 2 cycles, $76 \%$ of lesions resolved.

Using this tool, the authors demonstrate how standardized scoring can be used to demonstrate responses to therapy. Here they used MRI as a readout to gauge response to pamidronate in 32 patients with $\mathrm{CNO}$ who had been treated with pamidronate. While the study was retrospective and the treatment approach was not fixed, it provides additional data supporting the effectiveness of pamidronate in the treatment of $\mathrm{CNO}$. Although this study demonstrated good interreader reliability for the WB-MRI scoring tool, having only 2 readers and both from the same institution limits the generalizability of the study and further validation at other centers will be needed. Despite this, Panwar, et al ${ }^{14}$ have done an excellent job in laying the foundation for further investigation, and as CRMO monitoring practices become more standardized, further investigation and evaluation of treatment efficacies can be performed to develop stronger, data-driven treatment regimens.

\section{REFERENCES}

1. Giedion A, Holthusen W, Masel LF, Vischer D. Subacute and chronic "symmetrical" osteomyelitis]. [Article in multiple languages] Ann Radiol 1972;15:329-42.

2. Hedrich CM, Hahn G, Girschick HJ, Morbach H. A clinical and pathomechanistic profile of chronic nonbacterial osteomyelitis/ chronic recurrent multifocal osteomyelitis and challenges facing the field. Expert Rev Clin Immunol 2013 Sep;9:845-54.

3. Wipff J, Costantino F, Lemelle I, Pajot C, Duquesne A, Lorrot M, et

Table 1. A suggested whole-body MRI protocol for screening and monitoring CRMO.

\begin{tabular}{|c|c|c|c|c|c|c|c|}
\hline Plane & TR/TE, ms & $\begin{array}{c}\text { Slick Thickness/ } \\
\text { Space, } \mathrm{mm}\end{array}$ & $\mathrm{FOV}, \mathrm{cm}^{\mathrm{a}}$ & Matrix & $\begin{array}{c}\text { Acquisition } \\
\text { time }^{\mathrm{b}}, \mathrm{min}\end{array}$ & $\begin{array}{c}\text { Stations/ } \\
\text { Scans }\end{array}$ & $\begin{array}{l}\text { Total time } \\
\text { min }\end{array}$ \\
\hline Coronal whole-body 3D STIR & $4000 / 287$ & $4 / 0$ & 45 & $512 \times 307$ & $1: 37-2: 08$ & $4-5$ & $6: 28-10: 40$ \\
\hline Sagittal lower spine 3D STIR & $4000 / 289$ & $3 / 0$ & 40 & $384 \times 230$ & $1: 46$ & 1 & $1: 46$ \\
\hline Axial pelvis 3D STIR & $4000 / 291$ & $4 / 0$ & 40 & $384 \times 230$ & $2: 56$ & 1 & $2: 56$ \\
\hline Axial knees 3D STIR & $4000 / 292$ & $4 / 0$ & 40 & $384 \times 230$ & $2: 30$ & 1 & $2: 30$ \\
\hline Total time & & & & & & & $18: 58-23: 10$ \\
\hline
\end{tabular}

${ }^{a}$ Varies based on size. ${ }^{b}$ Time reflects usage on 1.5-Tesla (T) Aera, 1.5-T Avanto Fit, and 3-T Vida scanners (Siemens Healthcare) with total imaging matrix whole-body suite. CRMO: chronic recurrent multifocal osteomyelitis; FOV: field of view; MRI: magnetic resonance imaging; STIR: short-tau inversion recovery; TE: echo time; TR: repetition time. 
al. A large national cohort of French patients with chronic recurrent multifocal osteitis. Arthritis Rheumatol 2015;67:1128-37.

4. Zhao Y, Ferguson PJ. Chronic nonbacterial osteomyelitis and chronic recurrent multifocal osteomyelitis in children. Pediatr Clin North Am 2018;65:783-800.

5. Oliver M, Lee TC, Halpern-Felsher B, Murray E, Schwartz R, Zhao Y; CARRA SVARD CRMO/CNO workgroup. Disease burden and social impact of pediatric chronic nonbacterial osteomyelitis from the patient and family perspective. Pediatr Rheumatol Online J 2018;16:78.

6. Khanna G, Sato TS, Ferguson P. Imaging of chronic recurrent multifocal osteomyelitis. Radiographics 2009;29:1159-77.

7. Andronikou S, Kraft JK, Offiah AC, Jones J, Douis H, Thyagarajan $\mathrm{M}$, et al. Whole-body MRI in the diagnosis of paediatric CNO/ CRMO. Rheumatology 2020;59:2671-80.

8. Greer MC. Whole-body magnetic resonance imaging: techniques and non-oncologic indications. Pediatr Radiol 2018;48:1348-63.

9. Jurik AG, Klicman RF, Simoni P, Robinson P, Teh J. SAPHO and CRMO: the value of imaging. Semin Musculoskelet Radiol 2018;22:207-24.

10. Damasio MB, Magnaguagno F, Stagnaro G. Whole-body MRI: non-oncological applications in paediatrics. Radiol Med 2016;121:454-61.
11. Merlini L, Carpentier M, Ferrey S, Anooshiravani M, Poletti PA, Hanquinet S. Whole-body MRI in children: would a 3D STIR sequence alone be sufficient for investigating common paediatric conditions? A comparative study. Eur J Radiol 2017;88:155-62.

12. Darge K, Jaramillo D, Siegel MJ. Whole-body MRI in children: current status and future applications. Eur J Radiol 2008;68:289-98.

13. Zhao Y, Wu E, Oliver M, Cooper A, Basiaga M, Vora S, et al; Chronic Nonbacterial Osteomyelitis/Chronic Recurrent Multifocal Osteomyelitis Study Group and the Childhood Arthritis and Rheumatology Research Alliance Scleroderma, Vasculitis, Autoinflammatory and Rare Diseases Subcommittee. Consensus treatment plans for chronic nonbacterial osteomyelitis refractory to nonsteroidal antiinflammatory drugs and/or with active spinal lesions. Arthritis Care Res 2018;70:1228-37.

14. Panwar J, Tolend M, Lim L, Tse SM, Doria AS, Laxer RM, et al. Whole-body MRI quantification for assessment of bone lesions in chronic nonbacterial osteomyelitis patients treated with pamidronate: a prevalence, reproducibility, and responsiveness study. J Rheumatol 2021;48:751-9. 